\title{
Chiral Symmetry Breaking in QED
}

\section{with an External Field Varying in 3-d Space and Time}

D. G. Caldi and S. Vafaeisefat

ABSTRACT

We study quantum electrodynamics in an external field of gaussian configuration varying in all four dimensions and find that for certain ranges of the widths there is a non-zero value for $\langle\bar{\psi} \psi\rangle_{\text {ext }}$. This indication of chiral symmetry breaking in QED via a varying external field configuration which is quite similar to those in the GSI experiments, provides further evidence that the peaks seen in those experiments may be explained by a "new" phase of QED. 
In previous work [1] we have found that the expectation-value of $\Psi \psi$ in quantum electrodynamics (QED), evaluated in certain background classical electromagnetic field configurations, is non-zero. This is a signal of spontaneously broken chiral symmetry, and so, of a phase of QED which is different from the usual perturbative one. Besides being of interest for its own sake as a new aspect of QED and a new way of inducing chiral symmetry breaking in a field theory, this observation is also important for the scenario which we [2] have proposed as the explanation for the anomalous peaks seen in the low-energy heavy-ion collision experiments [3] at GSI, now being repeated and improved at Argonne and also at GSI. The foundation of our explanation is that the large and rapidly varying background electromagnetic fields generated in the ion collisions, can induce a rearrangement of the $\mathrm{QED}$ vacuum so that there is a metastable region where a new phase of QED exists. An important characteristic of this new phase is spontaneous chiral symmetry breaking, so that a new scale is generated, giving the possibility of electron-positron bound states in the range of $1.7 \mathrm{MeV}$. Motivated by this scenario, as well as for its own sake, we have been searching for direct evidence that background fields, similar, if possible, to those in the experiments, could cause spontaneous chiral symmetry breaking.

In our previous work [1] we found such positive evidence for the case of an external electric field with a gaussian shape in time and one spatial direction. We report here on the more realistic case of an external electric field with gaussian 
configuration giving variation in three-dimensional space as well as in time. Again we have found that for certain ranges of the widths there is a non-zero value for $\langle\bar{\psi} \psi\rangle_{\text {ext }}$. These results are rather encouraging since this 4-d configuration is even closer to the ones in the experiments.

The framework for our calculations, as before, is the Schwinger proper-time effective lagrangian with an external electromagnetic field [4]. This treats the external field non-perturbatively as well as including all the one-loop fermionic effects, but it does not include dynamical photons. We have argued previously [1] that dynamical photons should not only not destroy the effects we are seeing without them, since the coupling is weak, but rather should lead to an enhancement of these chiral symmetry breaking effects by helping to stabilize, at least somewhat, the new vacuum. It is of interest in this regard that recent papers [5] claim that dynamical photons alone actually can support chiral symmetry breaking, but that the effect is indeed quite weak.

The Schwinger proper-time formalism turns a field-theoretic problem into a problem in quantum mechanics evolving in a fictitious so-called proper time. Thus the order parameter for spontaneous chiral symmetry breaking $\langle\bar{\psi} \psi\rangle$ is written as a proper-time integral of a quantum mechanical transition amplitude:

$$
\begin{gathered}
\langle\bar{\psi}(\mathrm{x}) \psi(\mathrm{x})\rangle \\
=\mathrm{m} \int_{0}^{\infty} \mathrm{d} \tau \exp \left(-\mathrm{m}^{2} \tau\right) \operatorname{tr}\left\langle x\left|\mathrm{e}^{-H \tau}\right| \mathrm{x}\right\rangle,
\end{gathered}
$$


where the hamiltonian $H$ governing evolution of $x_{\mu}(\tau)$ is $-\left(\gamma_{\mu} \pi_{\mu}\right)^{2}$, with $\pi_{\mu}=p_{\mu}$ $\mathrm{eA}_{\mu}$ ext . The signal, as usual, that chiral symmetry is spontaneously broken is that as $\mathrm{m} \rightarrow 0,\langle\bar{\psi} \psi\rangle_{\neq 0}$, and, as discussed in [6], this requires $Z(\tau) \equiv \operatorname{tr}\left\langle\mathbf{x}\left|\mathrm{e}^{-H \tau}\right| \mathrm{x}\right\rangle \approx(\tau)^{-\frac{1}{2}}$ as $\tau \rightarrow \infty$ : this is then the behaviour we seek. Two limiting cases are well-known: if there is no external $\mathrm{A}_{\mu}, Z(\tau)$ goes as $\tau^{-2}$, whereas for constant $F_{\mu \nu}$ chiral symmetry is unbroken.

Hence non-constant fields are the focus of our interest, but these are in general intractable analytically, so we have been using computer simulations. To this end, we express $Z(\tau)$ in the form of a path integral:

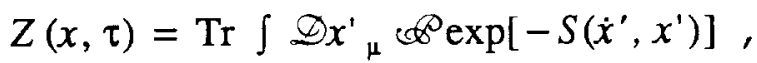

$$
\begin{aligned}
& S(\dot{x}, x)=\int_{0}^{\tau} \mathrm{d} \tau L\left[\dot{x}_{\mu}(\tau), x_{\mu}\left(\tau^{\prime}\right)\right], \\
& L(\dot{x}, x)=\frac{1}{4} \dot{x}_{\mu} \dot{x}_{\mu}+i e A_{\mu}(x) \dot{x}_{\mu}-\frac{1}{2} e \sigma_{\mu v} F_{\mu v}(x),
\end{aligned}
$$

with $x_{\mu}^{\prime}(0)=x_{\mu}^{\prime}(\tau)=x_{\mu}$. Because of various nonstandard aspects in the numerical 
evaluation of (2) we have been using a method which we developed [1, 7] from one designed [8] for complex actions, and this method is based on evaluating the density of states.

Expressing the action (without the matrix term) as a sum of its real and imaginary parts:

$$
S=\beta_{R} S_{R}(x)+\mathrm{i} \beta_{I} S_{I}(x),
$$

the density of states $\rho(E)$ is defined as

$$
\rho(E) \equiv \int \not x \quad \delta\left(S_{\mathrm{I}}-E\right) \exp \left(\beta_{\mathrm{R}} S_{\mathrm{R}}\right)
$$

where $E$ is the energy from the imaginary part of the action. The expectation value of an operator can then be expressed as

$$
\langle O(x)\rangle=\frac{\int \mathrm{d} E\langle O\rangle_{\mathrm{M}} \rho(E) \exp \left(\mathrm{i} \beta_{\mathrm{I}} E\right)}{\int \mathrm{d} E \rho(E) \exp \left(\mathrm{i} \beta_{\mathrm{I}} E\right)}
$$

where

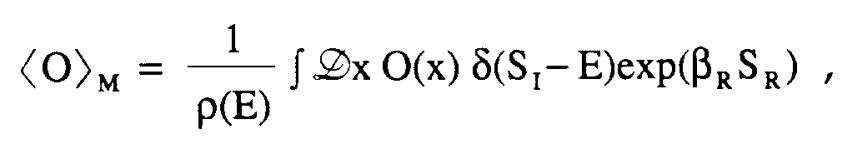


is the microcanonical average. What we need to calculate is the partition function $Z$ itself (2), hence for our purposes $O(x)$ is the matrix-valued term in the path integrand:

$$
O(x)=\operatorname{Tr} \oplus_{e x p}\left(-\frac{1}{2} \mathrm{e} \int_{0}^{\tau} \mathrm{d} \tau^{\prime} \sigma_{\mu \nu} \mathrm{F}_{\mu \nu}(\mathrm{x})\right)
$$

However, in general, this matrix term is complex, and we have found it more appropriate, as well as providing more stable results, to include the phase $\theta$ of this term with $S_{1}$, and only calculate the modulus in the microcanonical average. So finally the partition function is expressed most conveniently for our calculation as:

$$
\mathrm{Z}(\mathrm{e}, \mathrm{A}, \tau, \mathrm{x})=\mathrm{N}\langle\mathrm{O}(\mathrm{x})\rangle \tilde{\mathrm{Z}}(\mathrm{e}, \mathrm{A}, \tau)
$$

with

$$
\tilde{Z}(e, A, \tau)=\int \mathscr{D x} \exp \left(-\int_{0}^{\tau} d \tau^{\prime}\left(\frac{1}{4} \dot{x}_{\mu} \dot{x}_{\mu}+i e A_{\mu} \dot{x}_{\mu}+i \theta\right)\right),
$$

The factor $N$ in (10) gives an unimportant normalisation.

We calculate the density of states $\rho(E)$ according to the method we have described elsewhere $[1,7]$, by dividing the energy range into $N_{s}$ sets of $N_{b}$ bins and 
generating configurations weighted according to $\mathrm{P}(\mathrm{x}) \approx \exp \left[\beta_{\mathrm{R}} \mathrm{S}_{\mathrm{R}}(\mathrm{x})\right]$. We then calculate the imaginary energy of the configuration and record the event as a hit in the appropriate bin. We have successfully tested our method against the analytically known results (derived from Schwinger) for a constant field, which gives that $Z(\tau)$ goes as $\tau^{-1}$ and hence no chiral symmetry breaking.

But realistic fields are non-constant; unfortunately they are also almost always intractable to analytic treatment. Having had success with an electric field which has gaussian variation in two dimensions (with different behaviour in the euclidean temporal and spatial directions) reported in [1], we decided to try a field varying fully in all four dimensions. The external field we used is purely electric, i.e., $B=0$, and is of the form:

$$
E=E_{r}=e E \exp \left(-t^{2} / 2 W_{t}^{2}\right) \operatorname{sech}^{2}\left(r / W_{s}\right)
$$

This spherically symmetric, radial electric field is peaked at the origin in both the spatial and the temporal coordinates, where we have chosen the coupling $e$ times the maximum field strength $E$ to be equal to 1.0 . It should be noted that this fully four-dimensional field requires considerably more computer time than the twodimensional case we considered earlier. Furthermore, we have improved our method [7] and have employed larger lattices and have also included larger values of tau. All this further increased the necessary computer time. 
We first explored the parameter space for the temporal and spatial widths, $W_{t}$ and $W_{s}$, until we found that values around $W_{t}=1.0$ and $W_{s}=3.0$ gave the soughtfor $\tau-1 / 2$ fall-off for $Z$, as shown in the upper curve (with squares) and the lowest curve (with triangles) in Fig. 1. We looked at various values of the space-time point $x_{\mu}$ at which the expectation-value of $\Psi \psi$ is evaluated, in order to determine how large the region in space is where the new phase may be said to exist. More extensive exploration of this region (as well as the widths space) was possible with the simpler two-dimensional field [7], but here we have been able to confirm that the effect, which we initially found at $x_{\mu}=0.001$ (lowest curve, with triangles, in Fig. 1), persists to values of spatial $r$ around $1.6\left(x_{i}=0.95\right)$, where the E-field has been reduced to about $75 \%$ of its peak value (upper curve, with squares, in Fig. 1). This is also the region where the field is changing most rapidly. When $x_{i}$ was increased to about $1.5(r=2.65)$, where the field is about $50 \%$ of its maximum, the effect disappeared, as seen in the middle curve (with circles) in Fig. 1, with a tau fall-off of about 1.2 , closer to the constant field case, as might be expected in this region of smaller and slowly varying E-field.

The data in Fig. 1 were generated on a discretised proper-time circle ranging from 100 sites for tau=12 to 300 sites for tau=35. We typically used 55 sets of 8 bins each at tau=12 and increased this up to 80 sets of 8 bins each at tau=35. There were up to 200,000 hits per set, and 6 to 8 independent runs (in both directions in E) per tau-value were performed and averaged over (giving statistical errors in $\ln (\mathrm{Z})$ of less 
than $5 \%$ ) to obtain the data points in the figures.

We also looked at the question of whether both temporal and spatial variation in the field are necessary to obtain the desired square-root of tau fall-off. We first studied our field configuration (12) but without the gaussian variation in time. This time-independent 3 -d gaussian did not give $\tau^{-1 / 2}$ behaviour. We also considered a three-dimensional Coulomb field with gaussian variation in time and this also gave $\tau^{-1 / 2}$ behaviour; but when we made the field static, the fall-off changed to $1 / \tau$ at the same space-time point. Hence spatial variation of the external field, while necessary to achieve a non-zero $\langle\Psi \psi\rangle$, is not sufficient: temporal variation is also required. We have also explored the question of minimum field strength, but so far only with the simpler two-dimensional field configurations, and this will be reported elsewhere [7].

Besides studying in more detail various other aspects of this effect in the simpler two-dimensional case, we have also been examining a quite realistic field configuration produced by colliding charges. This also gives the desired $\tau^{-1 / 2}$ behaviour for $Z$ and will be reported in a future publication [9]. Hence it appears that various field configurations can give this signal of chiral symmetry breaking, at least in this formalism. What appears to be crucial is that the field vary relatively quickly in space and time, and that the observation point be in the region of rapid variation. Other methods of calculation are also being explored, with a view to having both a more inclusive system, as well as one in which, for example, one may 
study the existence, nature, and mass of the Nambu-Goldstone state here, the expected electropion.

It is a pleasure to thank A. Chodos for many stimulating discussions. The work was supported in part under DOE Grant No. DE-FG02-92ER40741. 


\section{REFERENCES}

1. D. G. Caldi and S. Vafaeisefat, Phys. Lett. B 287 (1992) 185.

2. D. G. Caldi and A. Chodos, Phys. Rev. D36, 2876 (1987); D. G. Caldi, A. Chodos, K. Everding, D. A. Owen, and S. Vafaeisefat, Phys. Rev. D39, 1432 (1989); L. S. Celenza, V. K. Mishra, C. M. Shakin, and K. F. Liu, Phys. Rev. Lett. 57, 55 (1986); L. S. Celenza, C. R. Ji, and C. M. Shakin, Phys. Rev. D36, 2144 (1987); Y. J. Ng and Y. Kikuchi, Phys.Rev. D36, 2880 (1987); Y. Kikuchi and Y. J. Ng, Phys. Rev. D38, 3578 (1988). For reviews, see D. G. Caldi, Comments Nucl. Part. Phys. 19, 137 (1989); and inVacuum Structure in Intense Fields, Proceedings of the NATO Advanced Study Institute, Cargèse, France, July, 1990, eds. H. M. Fried and B. Müller (Plenum, New York, 1991).

3. J. Schweppe et al., Phys. Rev. Lett. 51, 2261 (1983); M. Clemente et al., Phys. Lett. 137B, 41 (1984); T. Cowan et al., Phys. Rev. Lett. 54, 1761 (1985); T. Cowan et al., Phys. Rev. Lett. 56, 444 (1986); H. Tsertos et al., Phys. Lett. 162B, 273 (1985); H. Tsertos et al., Z. Phys. A 326, 235 (1987). For a review, see T. Cowan and J. Greenberg, in Physics of Strong Fields, ed. W. Greiner (Plenum, New York, 1987); see also the contributions of H. Bokemeyer and W. Koenig in the Cargèse 1990 Proceedings, op. cit.

4. J. Schwinger, Phys. Rev. 82, 664 (1951).

5. V. P. Gusynin, V. A. Miransky, and I. A. Shovkovy, Bogolyubov Institute for Theoretical Physics preprint, ITP-95-02E, February, 1995. 
6. T. Banks and A. Casher, Nucl. Phys. B169, 103 (1980).

7. D. G. Caldi and S. Vafaeisefat, in preparation.

8. A. Gocksch, Phys. Rev. Lett. 18, 2054 (1988); Phys. Lett. 206B, 290 (1988); G. Bhanot, A. Gocksch, and P. Rossi, Phys. Lett. 199B, 101 (1987); G. Bhanot, K. Bitar, and R. Salvador, Phys. Lett. 188B, 246 (1987); G. Bhanot, S. Black, P. Carter, and R. Salvador, Phys. Lett. 183B, 331 (1987); M. Karliner, S. Sharpe, and Y. F. Chang, Nucl. Phys. B302, 204 (1988).

9. D. G. Caldi and S. Vafaeisefat, in preparation. 


\section{Figure Caption}

Fig. 1. Data from simulations of $Z$ and fits, for gaussian E-field of Eq. (12), with $e E=1.0, \mathrm{~W}_{\mathrm{t}}=1.0, \mathrm{~W}_{\mathrm{s}}=3.0$. Upper curve (squares): $\mathrm{t}=0.0, \mathrm{r}=1.6$, fit gives $Z \propto \tau^{-0.52 \pm 0.03}$ with an $R^{2}=0.99$. Middle curve (circles): $t=0.0, r=2.65$, fit gives

$Z \propto \tau^{-1.22 \pm 0.06}$ with an $R^{2}=0.99$. Lowest curve (triangles): $t=0.001, r=0.0017$, fit gives $Z \propto \tau^{-0.51 \pm 0.04}$ with an $R^{2}=0.99$ 


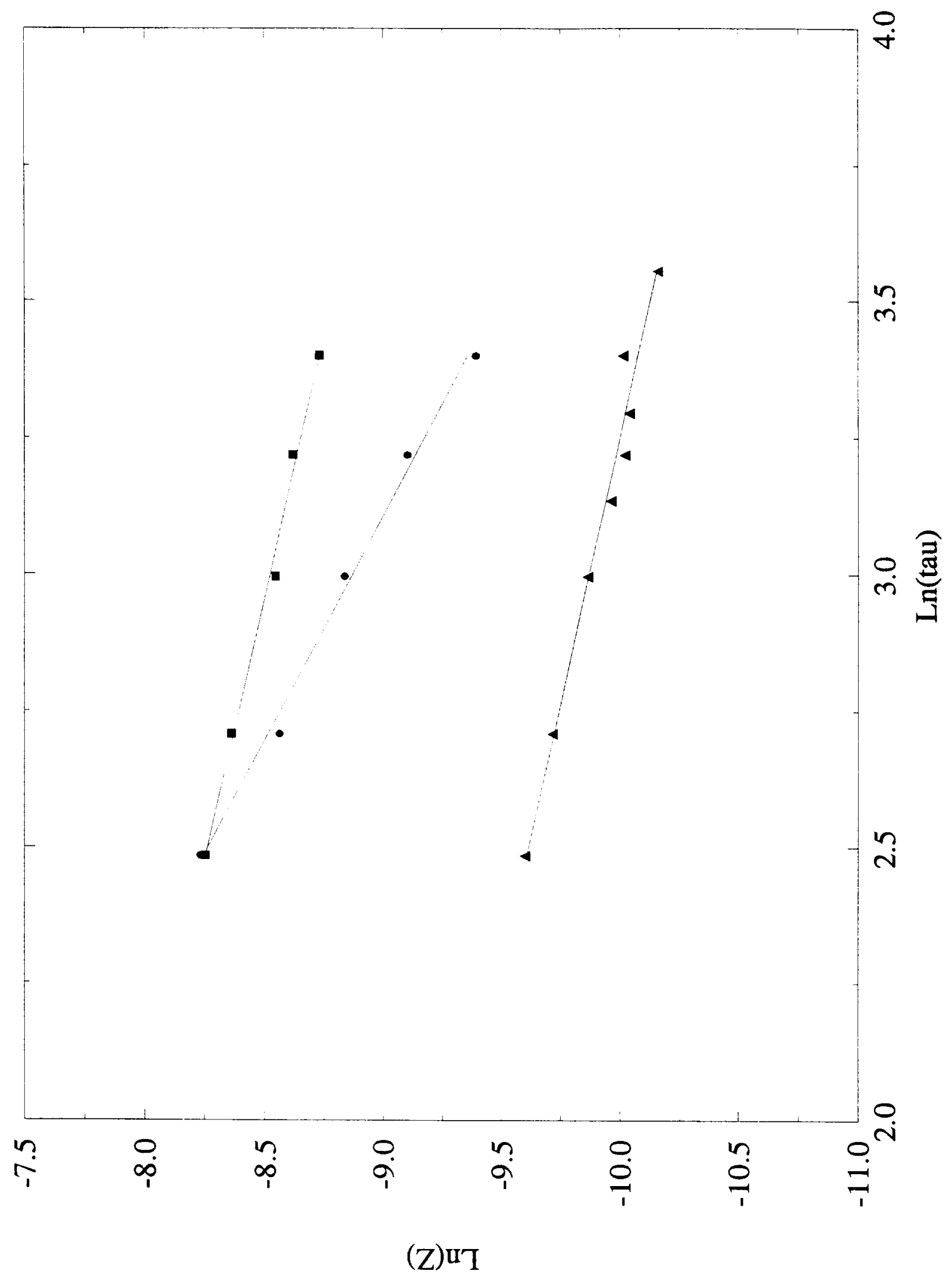

